\title{
Oligodendrocyte-Myelin Glycoprotein Is Present in Lipid Rafts and Caveolin-1-Enriched Membranes
}

\author{
MADANAMOHAN BOYANAPALLI, ${ }^{1}$ VICKY KOTTIS, ${ }^{2}$ OSCAR LAHOUD,${ }^{1}$ SAOUSSEN BAMRI-EZZINE, ${ }^{2}$ \\ PETER E. BRAUN, ${ }^{2}$ AND DANIEL D. MIKOL ${ }^{1 *}$ \\ ${ }^{1}$ Department of Neurology, University of Michigan, Ann Arbor, Michigan \\ ${ }^{2}$ Department of Biochemistry, McGill University, Montreal, Canada
}

KEY WORDS

axolemma; CNP; MO3.13; MOG; OMgp

\begin{abstract}
The oligodendrocyte-myelin glycoprotein is a ligand of the neuronal Nogo receptor and a potent inhibitor of neurite outgrowth, but its physiological function remains to be elucidated. The oligodendrocyte-myelin glycoprotein is anchored solely in the outer leaflet of the plasma membrane via its glycosylphosphatidylinositol anchor, and through its leucine-rich repeat domain, it likely interacts with other proteins. In the present study, we compare its buoyancy and detergent solubility characteristics with those of other myelin proteins. Based on its detergent solubility profile and membrane fractionation using established ultracentrifugation procedures, we conclude that the oligodendrocyte-myelin glycoprotein is a lipid raft component that is closely associated with the axolemma. Moreover, it associates with caveolin-1 and caveolin-1-enriched membranes. We postulate that, by virtue of its concentration in lipid rafts and perhaps through interactions with caveolin1 , the oligodendrocyte-myelin glycoprotein may influence signaling pathways. $\odot 2005$ Wiley-Liss, Inc.
\end{abstract}

\section{INTRODUCTION}

The oligodendrocyte (OLG)-myelin glycoprotein (OMgp) was initially identified as a glycosylphosphatidylinositol (GPI)-linked peanut agglutinin (PNA)-binding glycoprotein that appears relatively late in CNS development (Mikol and Stefansson, 1988). While it is believed to be myelin associated and paranodally localized (Mikol et al., 1990b), its exact cell(s) of origin is unclear. The structure of OMgp includes a highly conserved domain of eight tandem leucine-rich repeats (LRRs) and a Cterminal GPI anchor, both of which are likely to be of functional significance (Mikol et al., 1990a,b, 1993). The LRR domain is strongly implicated in mediating protein-protein interactions (Kobe and Deisenhofer, 1994, 1995), while the GPI lipid tail affords a high degree of lateral mobility and may facilitate biological responses through interactions with transmembrane signaling molecules (Brown, 1993; Sharom and Lehto, 2002).

GPI-linked proteins are often concentrated in lipid rafts in the vicinity of caveolae (Mayor et al., 1994; Schnitzer et al., 1995; Liu et al., 1997; Fielding and Fielding, 2003). Lipid rafts and caveolae are plasma membrane microdomains that contain abundant choles- terol, glycosphingolipids and signaling molecules, but are distinguished by the fact that only caveolae are enriched in caveolins (Lisanti et al., 1994b). Caveolins are small hairpin-shaped molecules that provide the structural backbone of caveolae vesicles (Lisanti et al., 1993) and have diverse effects on signal transduction (Okamoto et al., 1998). Caveolin-1 (Cav-1) [24 $(\alpha) \mathrm{kD}$ and $21(\beta) \mathrm{kD}$ isoforms], the most prevalent isoform, binds to itself to form a multimeric complex (Fra et al., 1995), serving as a scaffold for a variety of signaling molecules (Sargiacomo et al., 1995). Whether a component of lipid rafts or caveolae, GPI-linked proteins are thought to modulate signaling pathways through signaling complexes enriched in these membrane microdomains (Abrami et al., 2001; Massimino et al., 2002; Fielding and Fielding, 2003).

Myelin consists of a complex bioarchitecture that includes compact lamellae and looser membrane structures such as periaxonal membranes and paranodal loops (Trapp et al., 2004). While myelin serves an insulative function, it is also physiologically active and in close contact with the axons it ensheathes. In fact, rigorous myelin subfractionation procedures demonstrate copurification of myelin and axolemmal proteins, termed the myelin-axolemmal complex, reflecting high-affinity interactions (Menon et al., 2003). Within myelin, lipid raft domains are likely to compartmentalize signal transduction pathways that involve proteins such as $2^{\prime}, 3^{\prime}$-cyclic nucleotide $3^{\prime}$-phosphodiesterase (CNP) and myelin oligodendrocyte glycoprotein (MOG) (Kim and Pfeiffer, 1999). Here we compare the detergent solubility and membrane subfractionation profile of OMgp with that of other myelin proteins. We show that OMgp is a component of lipid rafts, in part colocalizes with and interacts with Cav-1, and is a constituent of the myelinaxolemmal complex, and we discuss implications for the possible physiological function of OMgp and its proposed function as an inhibitor of neurite growth (Kottis et al., 2002; Wang et al., 2002).

Grant sponsor: National Institutes of Health (NINDS); Grant number: NS4209901; Grant sponsor: Multiple Sclerosis Society of Canada.

*Correspondence to: Daniel D. Mikol, Department of Neurology, University of Michigan, Ann Arbor, MI 48109-0322. E-mail: dmiko@umich.edu

Received 15 December 2004; Accepted 18 April 2005

DOI 10.1002/glia.20237

Published online 20 June 2005 in Wiley InterScience (www.interscience. wiley.com). 


\section{MATERIALS AND METHODS Antibodies}

A mouse monoclonal antibody (mAb) and affinity-purified rabbit polyclonal antibody (pAb) against Cav-1 were purchased from Transduction Laboratories (C37120 and C13630, respectively; Lexington, KY). Rabbit anti-myelin-associated glycoprotein (MAG) pAb and rat anti-myelin basic protein (MBP) $\mathrm{mAb}$ were generous gifts from Dr. J. Salzer (New York University, New York, NY), and Dr. N. Groome (Oxford University, Headington, UK), respectively. An anti-CNP mAb was purchased from Sternberger Monoclonals (SMI91; Lutherville, MD), while antiNG2 pAb was obtained from Chemicon International (AB5320; Temecula, CA). Antibodies against voltage gated sodium channels, Caspr-1, and potassium channels (Kv1.2) were kindly provided by Dr. M. Rasband (University of Connecticut, Farmington, CT). Our rabbit anti-OMgp pAb was previously described (Mikol and Stefansson, 1988). In addition, we generated rabbit pAb against a multiple antigenic peptide (Posnett and Tam, 1989) of OMgp (OMgp-pep) that is 100\% conserved between mouse (Mikol et al., 1993) and human (Mikol et al., 1990b) (accession numbers Q63912 and X51694.1, respectively). OMgp-pep (LLDKSDTAYQWNLKYLDVSKN, residues 89-109) was generated in the University of Michigan Protein Structure Core Facility. Antigenicity analysis determined that OMgp-pep, contained within the LRR, was likely to be the most immunogenic region within OMgp. Antibodies against OMgp-pep were affinity-purified on an agarose-OMgp-pep column that was prepared using AffiGel 10 (Bio-Rad Laboratories, Hercules, CA).

\section{Cell and Tissue Sources}

Stripped adult rat brains or mouse brains were purchased from Pel-Freez (Rogers, AR). The human oligodendrocytic cell line M03.13 (McLaurin et al., 1995) was generously provided by Dr. N. Cashman (University of Toronto, Toronto, ONT, Canada). In general, cells were grown at $37^{\circ} \mathrm{C}$ in a humidified atmosphere with $10 \%$ $\mathrm{CO}_{2}$. Before analysis, MO3.13 cells were grown to $\sim 80 \%$ confluency.

\section{Detergent Extractions, Myelin Preparation, and Membrane Fractionation}

Rat brains placed in phosphate-buffered saline (PBS; $\sim 0.05 \mathrm{~g} / \mathrm{ml}$ ) plus $1 \mathrm{mM}$ phenylmethylsulfonylfluoride (PMSF) and protease inhibitor cocktail (BoehringerMannheim, Indianapolis, IN) with or without detergent at $4^{\circ} \mathrm{C}$ were disrupted using a Dounce homogenizer (6-8 strokes each loose pestle followed by tight pestle) and centrifuged at $100,000 \mathrm{~g}$ for $1 \mathrm{~h}$. Supernatant and pellet fractions were collected for analysis; the pellet fraction was further homogenized separately in one of three detergents: Triton X-100 (TX-100) at 1\% (vol/vol), octyl- glucopyranoside (OCTG) at $60 \mathrm{mM}$, and 3-[(3-cholamidopropyl)dimethylammonio]-1-propane sulfonate (CHAPS) at $30 \mathrm{mM}$. After centrifuging at $100,000 \mathrm{~g}$ for $1 \mathrm{~h}$, detergent soluble and insoluble fractions were collected for immunoblot analysis.

Compact myelin membranes were first prepared from rat brain by the standard method of Norton and Poduslo (1973) at $4^{\circ} \mathrm{C}$ in the presence of $1 \mathrm{mM}$ PMSF and protease inhibitor cocktail (Boehringer-Mannheim), and fractions were collected at different stages in the isolation procedure. In recent years, it has become apparent that distinct myelin compartments exist, and it has become possible to analyze myelin membranes in greater detail. Thus, in separate experiments, more comprehensive subfractions of mouse brain myelin were prepared according to the procedure of Menon et al. (2003), with all steps carried out at $4^{\circ} \mathrm{C}$. Here, P30 day-old mouse brains were dissected and homogenized in $0.32 \mathrm{M}$ sucrose $(5 \%$ w/v) using a Dounce homogenizer (15 strokes), and homogenates (18 ml) were layered over $0.85 \mathrm{M}$ sucrose $(18 \mathrm{ml})$ in each of four $40-\mathrm{ml}$ centrifuge tubes, which were then centrifuged at $140,000 \mathrm{~g}$ for $1 \mathrm{~h}$ in a Beckman SW28 rotor. Thirteen fractions were collected from gradient I: fraction $1(6 \mathrm{ml})$, fractions $2-12$ (2 ml each), fraction $13(7 \mathrm{ml})$, and a pellet. Fractions 7-9 (light band) and fractions 10-13 (dispersed band) were pooled and diluted with $10 \mathrm{mM}$ EGTA in water at $\mathrm{pH}$ 7.5. This sample was then homogenized (15 strokes) and centrifuged at $200,000 \mathrm{~g}$ for $20 \mathrm{~min}$ (Beckman $\mathrm{Ti}$ 50.2). Pellets were resuspended in $10 \mathrm{mM}$ EGTA ( $\mathrm{pH}$ 7.5), homogenized, stirred for $15 \mathrm{~min}$ and centrifuged at $35,000 \mathrm{~g}$ for $15 \mathrm{~min}$ (Sorvall SS-34). This was repeated once. The final pellets were resuspended in $18 \mathrm{ml}$ $0.85 \mathrm{M}$ sucrose and homogenized (15 strokes). A single discontinuous sucrose gradient was prepared with $2 \mathrm{ml}$ $1 \mathrm{M}$ sucrose, $18 \mathrm{ml} 0.85 \mathrm{M}$ sucrose/homogenate, $18 \mathrm{ml}$ $0.32 \mathrm{M}$ sucrose and centrifuged at $140,000 \mathrm{~g}$ for $90 \mathrm{~min}$. This constitutes gradient II.

Fourteen fractions were collected from gradient II: fraction $1(6 \mathrm{ml})$, fractions $2-12(2 \mathrm{ml}$ each $)$, fraction 13 $(8 \mathrm{ml})$, fraction $14(3 \mathrm{ml})$, and a pellet. Fractions $8-9$ (light density) and 10-13 (dispersed band) were combined and diluted with $2 \mathrm{mM}$ EGTA in water ( $\mathrm{pH} 7.5$ ), homogenized (15 strokes), and centrifuged at 200,000g for $20 \mathrm{~min}$. Pellets were resuspended in $120 \mathrm{ml} 2 \mathrm{mM}$ EGTA, homogenized (no stirring) and centrifuged at $35,000 \mathrm{~g}$ for $15 \mathrm{~min}$. Pellets were resuspended in $12 \mathrm{ml}$ $0.85 \mathrm{M}$ sucrose and homogenized. A single discontinuous gradient was prepared with $12 \mathrm{ml} 0.85 \mathrm{M}$ sucrose/homogenate, $12 \mathrm{ml} 0.75 \mathrm{M}$ sucrose, $12 \mathrm{ml} 0.32 \mathrm{M}$ sucrose and centrifuged at $140,000 \mathrm{~g}$ for $16 \mathrm{~h}$; 1 -ml fractions were collected and diluted with 2 vol of 2 mM EGTA ( $\mathrm{pH} 7.5$ ), and membranes were collected by centrifugation at $35,000 \mathrm{~g}$ for $15 \mathrm{~min}$. Pellets were resuspended in $0.5 \mathrm{ml}$ of $50 \mathrm{mM}$ Tris-HCl ( $\mathrm{pH}$ 7.5) plus protease inhibitor cocktail. Protein concentration was determined using a Protein Assay kit (Bio-Rad Laboratories); fractions 1-8 had very little detectable protein. Samples were pelleted by centrifugation and solubilized in $50 \mathrm{mM}$ Tris-HCl, $5 \%$ sodium dodecyl sulfate (SDS), $4 \mathrm{M}$ urea ( $\mathrm{pH}$ 6.8), and 
then mixed with sample buffer (Laemmli, 1970), and $5 \mu \mathrm{g} /$ well of protein $(2.5 \mu \mathrm{g} /$ well for CNP, MBP, Caspr-1) was loaded without boiling on $5 \%$ or $12 \%$ sodium dodecyl sulfate-polyacrylamide gel electrophoresis (SDS-PAGE) gels for immunoblot analysis.

Caveolin-enriched membranes (CEMs) were prepared from rat brain or MO3.13 cells by one of two discontinuous density-gradient centrifugation methods, as previously described (Mikol et al., 1999). While both methods take advantage of the buoyancy of CEMs, one also involves the use of nonionic detergent, given the detergent insolubility of CEMs (Lisanti et al., 1995); the other uses sonication without detergent (Song et al., 1996). Using either method, CEMs fractionate near the 1525\% sucrose interface (Mikol et al., 1999). Generally, the detergent-free method is preferred, as use of detergents can artifactually result in both the loss of protein association with CEMs, as well as the apparent concentration of other molecules, such as GPI-linked proteins (Schnitzer et al., 1995; Liu et al., 1997). Briefly, $\sim 0.75 \mathrm{~g}$ rat brain or two $150 \mathrm{~mm}$ plates of MO3.13 cells were used per 12-ml centrifuge tube, whether the detergentbased or detergent-free protocol was followed. In either case, after saving an aliquot of the initial homogenate, $6 \mathrm{ml}$ of lysate in $40 \%$ sucrose was placed in a $12-\mathrm{ml}$ centrifuge tube and overlaid sequentially with $2 \mathrm{ml}$ each $25 \%$ sucrose, $15 \%$ sucrose, and $5 \%$ sucrose, then centrifuged at $200,000 \mathrm{~g}$ for $18-20 \mathrm{~h}$. After centrifugation, 12 equal-volume (1-ml) aliquots from the top to bottom of the centrifuge tube were collected, as was the insoluble pellet, for immunoblot analysis.

\section{Immunoprecipitations, SDS-PAGE, and Immunoblotting}

For immunoblotting purposes, cell and tissue lysates were generally solubilized with RIPA ( $20 \mathrm{mM}$ Tris, 0.16 $\mathrm{M} \mathrm{NaCl}, 1 \mathrm{mM}$ EDTA, $1 \mathrm{mM}$ EGTA, $1 \%$ TX-100, $1 \% \mathrm{Na}$ deoxycholate, $0.1 \%$ SDS, $1 \mathrm{mM} \mathrm{Na}_{3} \mathrm{VO}_{4}[\mathrm{pH}$ 7.2]) + protease inhibitors. For immunoprecipitations, $60 \mathrm{mM}$ OCTG was used as the sole detergent, as this confers greater solubility to Cav-1 and Cav-1-associated proteins (Lisanti et al., 1995; Song et al., 1996). For immunoprecipitations, lysates were sonicated, centrifuged to remove particulates and insoluble membranes, and incubated for 30 min with protein A/G PLUS-agarose (Santa Cruz Biotechnology, Santa Cruz, CA). Soluble portions were mixed end-over-end overnight at $4^{\circ} \mathrm{C}$ with antibody to the protein of interest or pre-immune sera, and immune complexes were then precipitated with protein A/G PLUS-agarose for $2 \mathrm{~h}$. Brain lysates, MO3.13 cell fractions, washed immunoprecipitates or pre-stained molecular mass standards (Amersham Life Science, Arlington Heights, IL) were electrophoretically separated by SDS-PAGE, transferred at $100 \mathrm{~V}$ for $1 \mathrm{~h}$ onto Protran nitrocellulose membranes (Schleicher and Schuell, Keene, NH), and blocked overnight with $4 \%$ bovine serum albumin (for mAbs) or $5 \%$ nonfat powdered milk (for pAbs) in TBST ( $10 \mathrm{mM}$ Tris- $\mathrm{HCl}, 150 \mathrm{mM}$
$\mathrm{NaCl}, 0.05 \%$ Tween-20). Membranes were incubated with the primary antibody of interest in TBST overnight, washed $3 \times 10 \mathrm{~min}$ in TBST, incubated for $1 \mathrm{~h}$ with horseradish peroxidase-conjugated secondary antibody (Santa Cruz Biotechnology), again washed extensively, and finally visualized with enhanced chemiluminescence (ECL) reagents (Amersham, Piscataway, NJ). Parallel immunoblots were incubated in the absence of primary antibody (conjugate controls), and positive and negative control lysates were immunoblotted along with test lysates in order to verify specific binding.

\section{RESULTS}

While OMgp, MAG, and MBP are all CNS myelin proteins, they are differentially distributed in myelin membrane fractions. As shown in Figure 1, using a standard method of myelin membrane preparation from rat brain, OMgp is much more enriched in the intermediate osmotic shock fraction, which contains primarily loose myelin membranes and myelin fragments, as well as membranes from other cell types, as compared with the final preparation, which contains predominantly compact myelin membranes (Norton and Poduslo, 1973). By comparison, MBP is enriched in compact myelin and MAG is found in both compartments to a roughly equal extent as compared with the total brain homogenate. These biochemical findings indicate that the three myelin proteins are distributed preferentially in different membrane domains.

OMgp, MAG, and MBP also differ in terms of their buffer and detergent solubilities. OMgp, a GPI-linked myelin protein, almost exclusively partitions in the membrane fraction of rat brain (Fig. 2), in keeping with earlier studies of human brain OMgp (Mikol and Stefansson, 1988). A very small fraction of OMgp is soluble, which presumably reflects protein released by endogenous phosphatidylinositol-specific phospholipase-C. For comparison purposes, Cav-1 was examined. It is insoluble in buffer, consistent with its membrane structure. MBP is also insoluble in buffer, reflecting its behavior as an extrinsic protein tightly bound by ionic interactions that require high ionic strength to disrupt (Smith and Braun, 1988). A small amount of MAG is soluble in buffer alone, is slightly smaller than full-length MAG, and likely represents proteolytically released dMAG (Sato et al., 1984). The $100,000 \mathrm{~g}$ insoluble membrane pellet was next separately homogenized in three nonionic detergents: TX-100, OCTG, and CHAPS. OCTG and CHAPS structurally resemble glycosphingolipids and cholesterol, respectively, and have been found to solubilize GPI-linked proteins better than many nonionic detergents (Hooper and Turner, 1988). As shown in Figure 2, OMgp remains insoluble in TX-100 despite the fact that its buoyancy characteristics change in the presence of this detergent (see Fig. 6). OMgp is quite soluble in OCTG, as previously noted (Kottis et al., 2002) and, to a slightly lesser extent, in CHAPS. Of note, OMgp TX-100 solubility increases when membranes are 

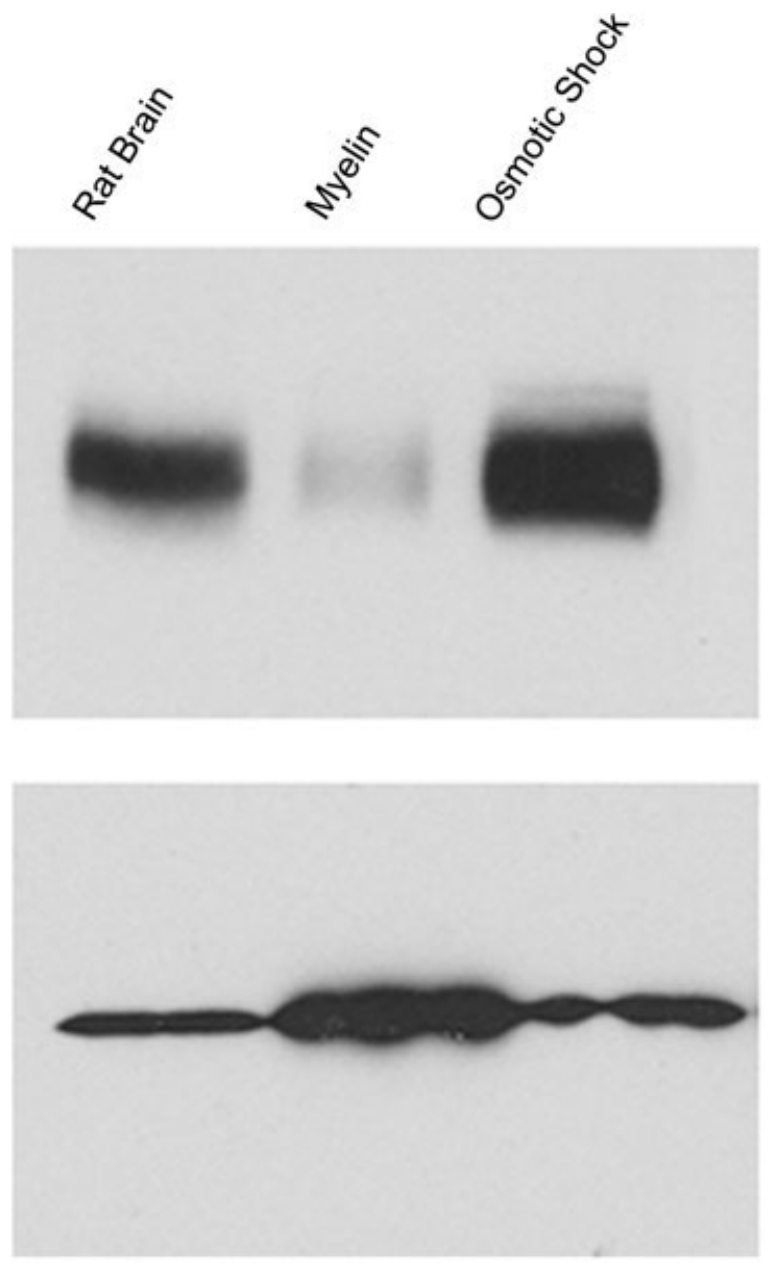

MBP

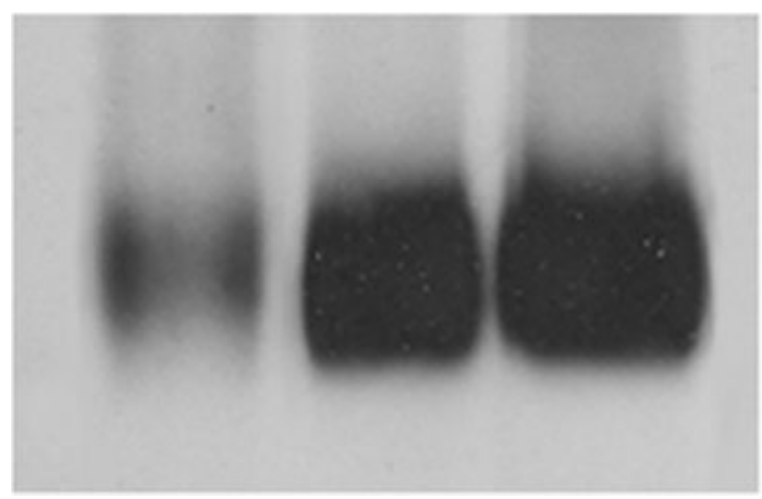

MAG

Fig. 1. Myelin protein enrichment within various membrane compartments from rat brain. Equal-volume aliquots of rat brain homogenate, purified myelin from rat brain or the osmotic shock fraction from a myelin preparation were immunoblotted with anti- oligodendrocyte (OLG)-myelin glycoprotein (OMgp), anti-MBP, and anti-MAG. OMgp is not enriched in the compact myelin fraction, whereas MBP and MAG are. OMgp is relatively enriched in the osmotic shock fraction, which contains looser myelin membranes, myelin fragments and membranes derived from other neural cells. These results emphasize that the three myelin proteins are contained within different membrane domains.

incubated at $37^{\circ} \mathrm{C}$, which is a feature of lipid raft membranes (not shown) (Kim and Pfeiffer, 1999). By comparison, MBP is at least equally soluble in TX-100 as in

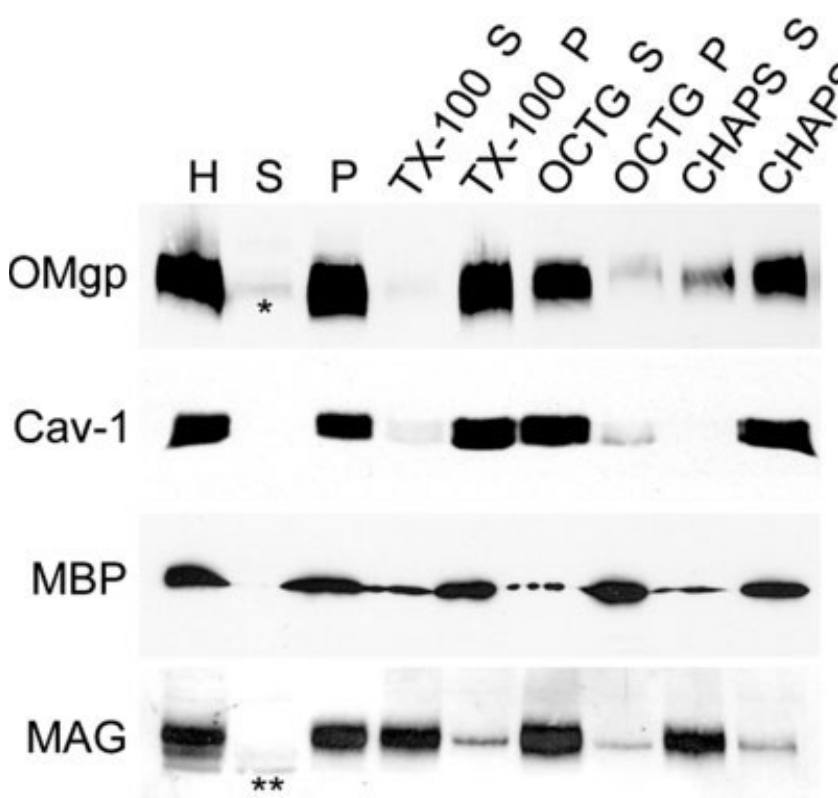

Fig. 2. Buffer and detergent extracts of rat brain. Rat brain homogenate $(\mathrm{H})$ was homogenized in buffer and centrifuged at 100,000gfor $1 \mathrm{~h}$. Equal volume aliquots of the supernatant $(\mathrm{S})$ and pellet $(\mathrm{P})$ were separated by SDS-PAGE. Very small fractions of oligodendrocyte (OLG)-myelin glycoprotein (OMgp) (asterisk) and MAG (double asterisk) are soluble in buffer alone. The insoluble pellet was next homogenized in either TX-100, OCTG, or CHAPS, and supernatants (S) and pellets $(\mathrm{P})$ of each were also separated by SDS-PAGE. Samples were immunoblotted with anti-caveolin-1 (Cav-1), anti-OMgp, anti-MAG, or anti-MBP. Cav-1 and OMgp have similar solubility characteristics: both are largely insoluble in TX-100 and highly soluble in OCTG, while OMgp is slightly more soluble in CHAPS. MAG and MBP appear roughly equally soluble in all three detergents. Of note, MBP isoforms are not separated on this 5\% acrylamide gel, such that MBP appears as a single band.

OCTG or CHAPS. The detergent solubility characteristics of MAG are similar to those of MBP, in that both are roughly equally soluble in all three detergents, although MAG is generally more detergent soluble. The detergent solubility profile of Cav-1 is similar to that of OMgp, with the exception that OMgp is more soluble in CHAPS than is Cav-1. These findings support the view that OMgp has solubility characteristics distinct from MAG and MBP.

We then carried out more definitive experiments to determine whether OMgp is contained within lipid raft membranes of myelin, using established procedures that begin with extraction of mouse myelin (Kim and Pfeiffer, 1999). First, rat brain myelin was centrifuged in sucrose gradients after TX-100 extraction. Immunoblot analysis of fractions shows that OMgp, like CNP, floats to the lower density fractions of the sucrose gradient (Fig. 3A). Purified mouse myelin was also extracted with TX-100 into insoluble and soluble fractions (Fig. 3B). As is the case for CNP, a subpopulation of OMgp is insoluble in TX-100. OMgp from purified myelin is relatively more TX-100 soluble as compared with whole brain extraction (see Fig. 2), which likely reflects the fact that more manipulative steps have been carried out in first obtaining myelin, and that the membrane environment of 

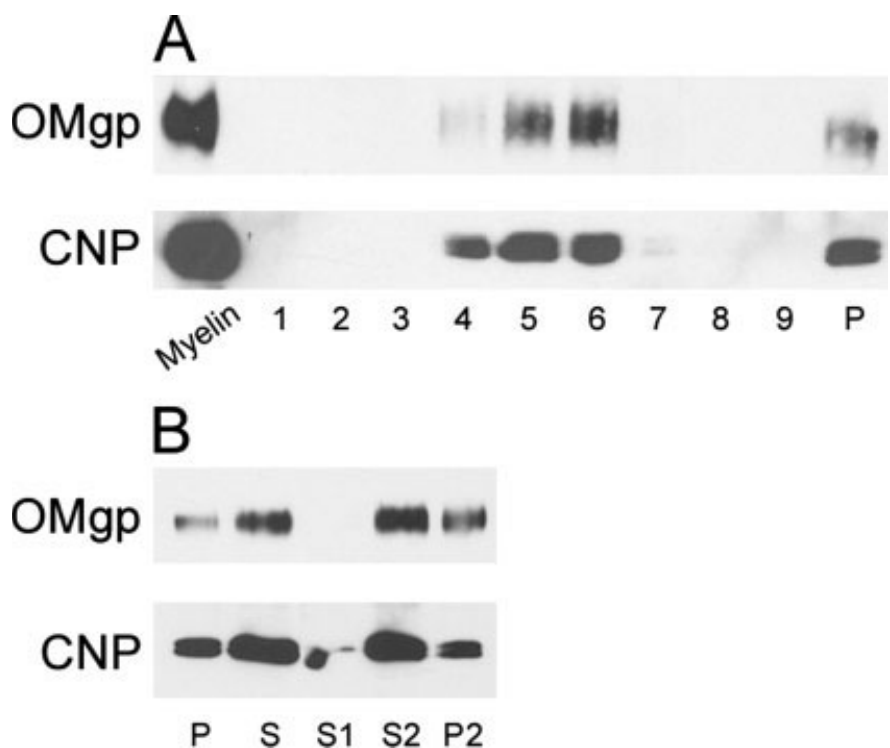

Fig. 3. Identification of oligodendrocyte (OLG)-myelin glycoprotein (OMgp) as a DIGCEM-associated protein. A: Total rat brain myelin was floated in sucrose gradients after TX-100 extraction at $4^{\circ} \mathrm{C}$. Nine fractions and pellet were collected and immunoblotted for OMgp and CNP. Immunoblot analysis shows that OMgp, like CNP, a DIGCEM-associated protein, floats to the lower-density fractions (4-6) in the sucrose gradient. B: Purified mouse myelin was extracted with TX-100 at $4^{\circ} \mathrm{C}$ and centrifuged to separate into detergent insoluble pellet $(\mathrm{P})$ and detergent soluble supernatant (S) fractions. As for CNP, a certain percentage of OMgp is found to be insoluble in TX-100. Pretreatment of purified myelin with $0.2 \%$ saponin at $4^{\circ} \mathrm{C}$ followed by centrifugation separated a soluble fraction $\mathrm{S} 1$ from an insoluble fraction that was further extracted with TX-100 at $4^{\circ} \mathrm{C}$ and centrifuged into detergentsoluble supernatant S2 and insoluble pellet (P2). Both OMgp and CNP are found in supernatant S2, a requirement of DIGCEM-associated proteins.

OMgp in isolated myelin differs somewhat from that in whole brain. In any case, the solubility pattern seen here for both OMgp and CNP is similar to that observed by Kim and Pfeiffer for CNP (1999). Finally, pretreatment of purified myelin with $0.2 \%$ saponin at $4{ }^{\circ} \mathrm{C}$, followed by centrifugation, generated an insoluble fraction that was further extracted with TX-100 at $4{ }^{\circ} \mathrm{C}$ and centrifuged to yield a detergent soluble supernatant (S2) containing both OMgp and CNP, and a pellet (P2) partially depleted of OMgp and CNP. This latter observation is in accord with the report of Kim and Pfeiffer (1999), except that in our hands, using mouse myelin, the depletion of OMgp and CNP was not as complete as from rat myelin. Thus OMgp is associated with detergent-insoluble, glycosphingolipid/cholesterol-enriched microdomains (DIGCEM) and is a raft-associated protein.

We sought to dissect the membrane localization of OMgp further by preparing fractions according to Menon et al. (2003). We included in our marker profile Cav-1 and NG2, the latter being a proteoglycan expressed by glial progenitor cells (Chang et al., 2000; Dawson et al., 2003; Aguirre et al., 2004). In the absence of quantification data for individual proteins, it is still possible to interpret the profile visually by comparing band intensities amongst fractions, given that each lane

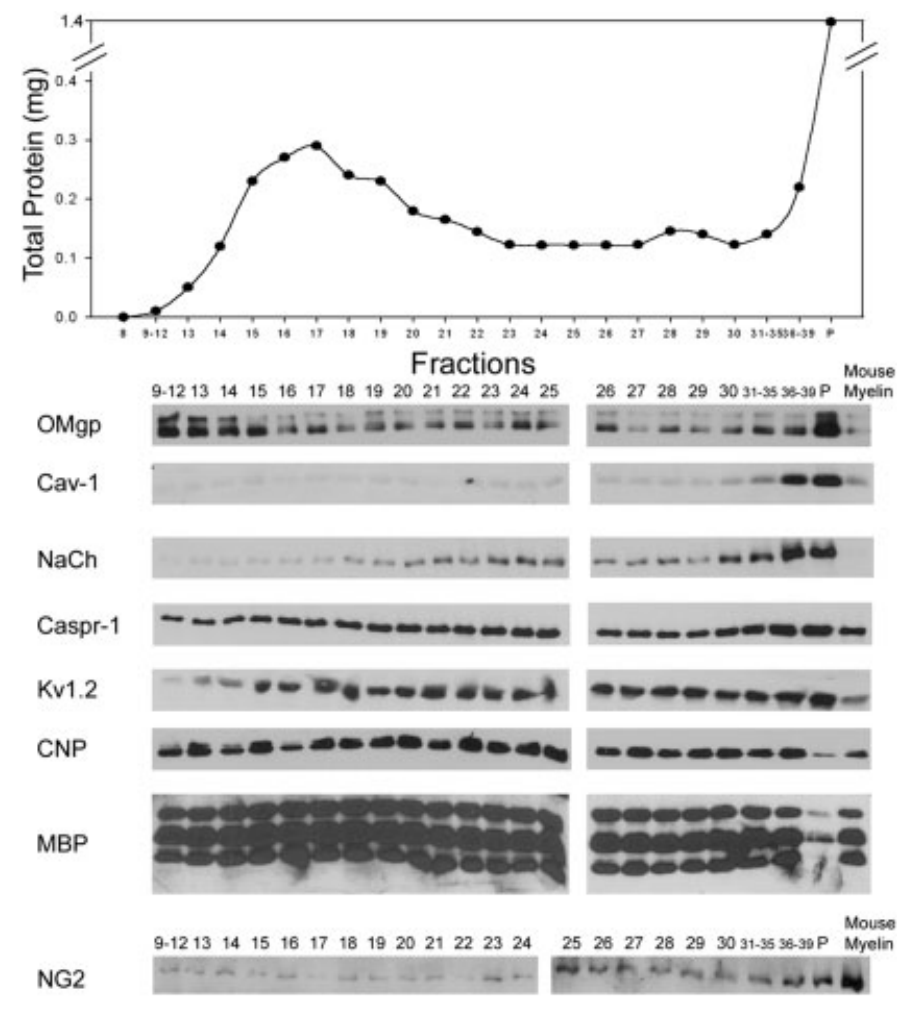

Fig. 4. Subfractionation of mouse myelin membranes. Mouse brain myelin was subfractionated as described in Materials and Methods. Oligodendrocyte (OLG)-myelin glycoprotein (OMgp) is detected in every subfraction of the "myelin" preparation, but is relatively enriched (largest band) in the pellet at the bottom of the gradient, which is enriched in axolemma elements. Protein content is recorded in the protein profile of the gradient. These data are a typical set taken from four independent experiments.

represents a constant amount of protein taken from the fractions. As shown in Figure 4, OMgp is present to some extent in every subfraction of the myelin preparation, but it is relatively most abundant in the pellet, which is enriched in Caspr-1 and sodium and potassium channels. These results reinforce those of Menon et al. (2003), in that axolemmal components are retained in purified "myelin" and demonstrate that OMgp is a component of the myelin-axolemmal complex, or possibly of OMgp-associated membranes that adhere to this complex. Likewise, NG2-associated membranes bind to myelin-axolemmal components, an observation not previously noted. As for OMgp and the sodium channel, NG2 is more concentrated in the very dense and pellet fractions.

To investigate partitioning of OMgp in Cav-1-enriched membranes, CEMs were prepared from rat brain using either the TX-100 (Fig. 5A) or detergent-free sodium carbonate method (Fig. 5B), or from MO3.13 cells using the sodium carbonate method (Fig. 5C), as detailed in the Methods. Using either method, buoyant CEMs partition near the 15\%/25\% sucrose interface (Lisanti et al., 1995; Song et al., 1996). OMgp is highly enriched within rat brain CEMs prepared by the TX-100 method, which in part might reflect artifactual partitioning of a GPI-linked 


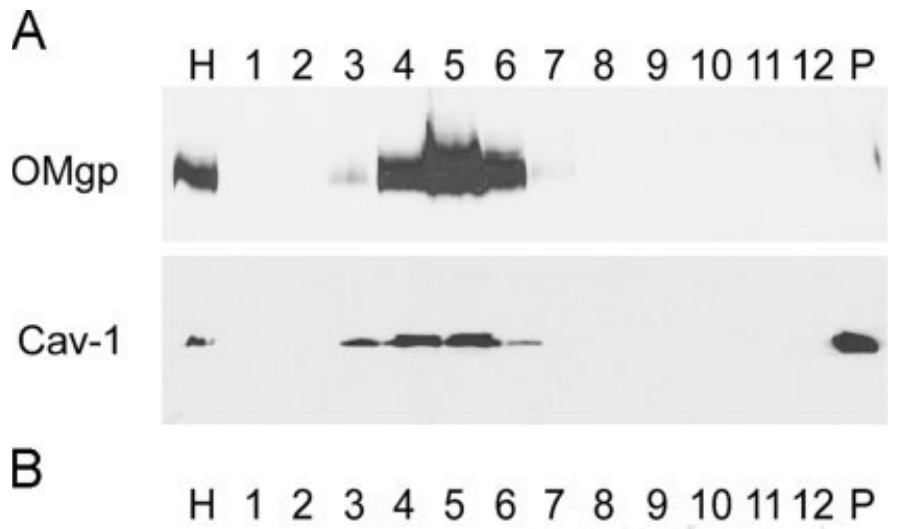

омер 'म

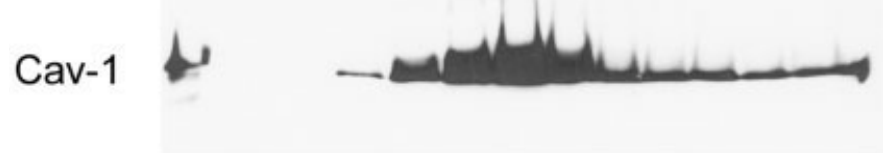

C

H $1 \begin{array}{llllllllll} & 2 & 3 & 4 & 5 & 6 & 7 & 8 & 9 & 101112 \mathrm{P}\end{array}$

OMgp
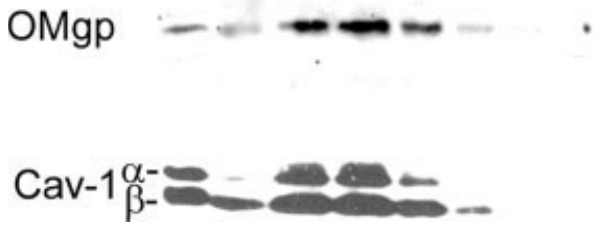

Fig. 5. Oligodendrocyte (OLG)-myelin glycoprotein (OMgp) is contained within caveolin-enriched membranes (CEMs) from rat brain or MO3.13 cells. CEMs were prepared from rat brain by either the TX-100 (A) or detergent-free (B) methods (fractions 1, 2: $5 \%$ sucrose; fractions 3, 4: $15 \%$ sucrose; fractions 5, 6: $25 \%$ sucrose; fractions 7-12: $40 \%$ sucrose). As shown in A, OMgp and caveolin-1 (Cav-1) are enriched in CEMs using the TX-100 method. Using the detergent-free method, the bulk of Cav-1 and a fraction of OMgp are detected in CEMs near the $15 \% / 25 \%$ sucrose gradient (B). OMgp appears to be more buoyant in the presence of TX-100, but nonetheless a fraction of OMgp is contained within detergent-free CEMs, as shown by its presence in fractions 4-6. Using the detergent-free method of preparing CEMs, OMgp is highly enriched in CEMs from MO3.13 human oligodendrocytic cells (C) OMgp, as detected by anti-OMgp-pep, is present in the same fractions as Cav-1. On this high percentage (15\%) acrylamide gel, Cav-1 separates into its two isoforms, $24 \mathrm{kD}(\alpha)$ and $21 \mathrm{kD}(\beta)$.

protein within lipid rafts in the presence of TX-100 (Schnitzer et al., 1995). Using the detergent-free method of CEM preparation, OMgp is largely in fractions 7-12 (40\% sucrose layer), although a smaller fraction is clearly contained within CEMs (Fig. 5, lanes 4, 5, and 6). These data show that a subpopulation of OMgp associates with membranes enriched in Cav-1 and suggest indirectly that OMgp is highly mobile, given that its average buoyancy differs considerably in the presence/absence of detergents. We speculate that localization of OMgp in Cav-1-enriched and nonenriched lipid raft domains is dynamic and changes in response to as yet unknown biological triggers. It is well recognized that protein association with

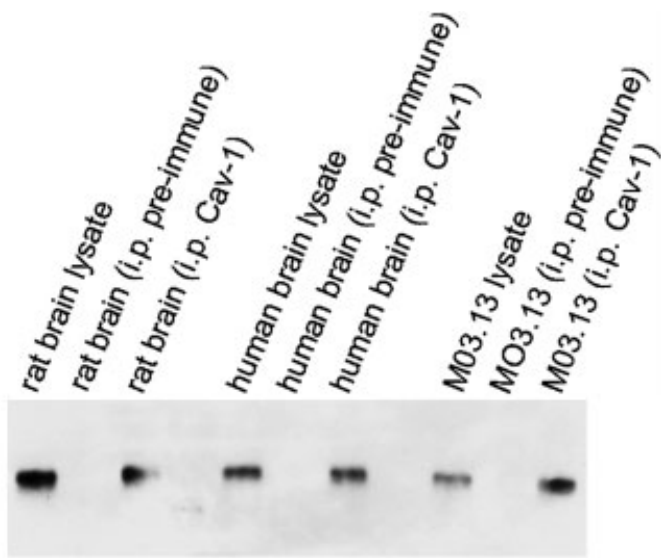

Fig. 6. Oligodendrocyte (OLG)-myelin glycoprotein (OMgp) and caveolin-1 (Cav-1) co-immunoprecipitate. Human brain, rat brain or MO3.13 lysates $(100 \mu \mathrm{g}$ each), or lysates $(500 \mu \mathrm{g}$ each) first immunoprecipitated (i.p.) with anti-Cav-1 pAb or preimmune serum, were immunoblotted with anti-OMgp-pep and the total i.p. analyzed. As shown, OMgp is immunoprecipitated with Cav-1 but not with rabbit preimmune serum. The lysates serve as positive controls for OMgp immunoblotting. These results were reproduced in four separate experiments.

Cav-1-enriched domains may be a dynamic process (Mineo et al., 1999; Chapman et al., 1999).

The human OLG-related cell line M03.13 expresses OMgp and other myelin proteins such as MBP (McLaurin et al., 1995) but does not make myelin. As shown in Figure 5C, OMgp is enriched within CEMs of MO3.13 cells using the detergent-free method. Here Cav-1 appears as a doublet due to the higher gel concentration (15\% acrylamide); when using lower concentrations ( $<10 \%$ acrylamide), Cav- 1 is generally seen as a single band (Mikol et al., 2002). The above results indicate that OMgp associates with Cav-1-enriched membranes, but does not imply direct or indirect interactions between the two proteins. Using MO3.13 cell lysates and brain homogenates, we show that OMgp co-immunoprecipitates with Cav-1 (Fig. 6). Given that both Cav1 and OMgp are highly soluble in OCTG, this detergent was utilized for immunoprecipitations. This association with Cav-1 is likely to be indirect in view of the anchorage of Omgp in the outer leaflet of the plasma membrane and suggests that OMgp associates with the Cav1 complex through intermediary proteins or lipids.

\section{DISCUSSION}

Glycosphingolipids, cholesterol, and GPI-linked proteins cluster together in distinct domains of the outer leaflet of the plasma membrane, termed lipid rafts, in which specific proteins are included while others are excluded. Lipid rafts are characterized by insolubility in certain detergents such as TX-100 at $4^{\circ} \mathrm{C}$ (Schroeder et al., 1994; Ahmed et al., 1997), and it is now clear that such microdomains exist in living cells (Kurzchalia and Parton, 1999), including OLGs (Taylor et al., 2002), where their functions are thought to include concen- 
tration of signaling complexes and protein trafficking (Simons and Wandinger-Ness, 1990; Brown and London, 1998; Simons and Toomre, 2000). Our findings support the view that OMgp exists in a unique lipid raft environment.

Fractionation of OMgp during myelin purification and detergent solubility profile differ from two other myelin proteins, MAG and MBP (Figs. 1 and 2). Moreover, using several criteria, OMgp is found in DIGCEM or lipid rafts (Figs. 3 and 4). Although we have not used their full complement of membrane markers, we have reproduced the observations of Menon et al. (2003) regarding the myelin and axolemmal proteins that are normally present in conventional mouse myelin preparations, for which "myelin purity" is often assumed. OMgp is present to some extent in every subfraction of the preparation, but it is relatively abundant in the pellet at the bottom of the gradient, where the total protein content comprises $25 \%$ of the protein applied to this gradient. In other words, a significant amount of OMgp is associated with membrane elements that are relatively depleted of the myelin proteins MBP and CNP but enriched in elements of the axolemma (voltage-gated sodium channels; Caspr-1; potassium channels).

Given the paucity of lamellar myelin components in the pellet, these findings indicate that OMgp is either present in the axolemma or in membrane elements of oligodendroglial origin that remain tightly associated with the axolemma, consistent with the observations of Menon et al. (2003), or both. The cellular origin(s) of OMgp are ambiguous. OMgp was originally named for its expression by OLGs and concentration within myelin membranes (Mikol and Stefansson, 1988; Habib et al., 1998b). It may be expressed to some extent by neurons as well (Habib et al., 1998b), but we have been unable to detect it in cultured neurons (mouse cerebellar granule cells, anterior horn cells; P.E. Braun and D.D. Mikol, unpublished observations). Further work is needed to clarify the degree of OMgp expression by different neural populations, particularly during development, as pointed out in a recent review on OMgp (Vourc'h and Andres, 2004).

Enrichment of Cav-1 in the pellet of the myelin subfractionation procedure (Fig. 4), a component of specialized membrane domains related to "lipid rafts," is concordant with our data showing that OMgp associates with caveolar and lipid raft membranes. OMgp is found in all subfractions, but is enriched where the "lightest" myelin elements occur (fractions 9-14) with the lowest protein content, and where there is the lowest abundance of (1) $\mathrm{Na}^{+}$channels; (2) $\mathrm{K}^{+}$channels; and (3) Cav1 . This may reflect the presence of OMgp in membranes that are not part of the myelin organelle, but that adhere to it. NG2 is not a protein of myelinating OLGs, but rather of glial progenitor cells, and it does not appear to associate as well with lamellar myelin components as with membranes in the pellet. The finding of Cav-1 in the myelin-axolemmal complex is not surprising, in that Cav-1 is known to regulate diverse signaling molecules. It is likely that Cav-1 is localized in part to the paranodal membranes, which is in keeping with our findings from peripheral nerve myelin (Mikol et al., 1999).

Cav-1 has previously been identified in cultured OLGs and myelin sheets (Arvanitis et al., 2004). Using either method of CEM preparation, Cav-1 and OMgp are contained in buoyant membranes from rat brain and MO3.13 cells (Fig. 5). CEMs share many of the features of caveolae (Lisanti et al., 1995; Song et al., 1996), including enrichment in caveolin, cholesterol, glycosphingolipids, and a variety of signal-transducing components (Sargiacomo et al., 1993; Lisanti et al., 1994b). Caveolae do not equate with lipid rafts, but can be considered a subset of them (Hooper, 1999; Kurzchalia and Parton, 1999; Fielding and Fielding, 2003). In the presence of caveolin, these membrane domains can invaginate to form uniformly sized (50-100-nM) vesicles (Okamoto et al., 1998). A "caveolae signaling hypothesis" proposes that compartmentalization of signaling molecules within caveolae allows for efficient coupling of activated receptors to a number of different effector systems (Sargiacomo et al., 1993; Lisanti et al., 1994a; Okamoto et al., 1998). These data support the view that, within the OLG membrane, OMgp exists in an environment rich in signaling complexes.

OMgp is present in CEMs and does, at least in part, co-immunoprecipitate with Cav-1 from MO3.13 cells (Fig. 6). Interestingly, using the detergent-free method, OMgp is more enriched in CEMs from MO3.13 cells than rat brain. We hypothesize that OMgp is closely associated with OLG lipid rafts, but that its association with Cav-1-enriched membranes might vary depending upon a variety of factors, including degree of Cav-1 expression, cellular metabolic state, level of differentiation, and degree of OMgp clustering (through interactions with other molecules). We have previously shown that Cav-1 expression by Schwann cells increases during development and decreases following nerve transection (Mikol et al., 1999, 2002), which is consistent with findings in non-neural cell types that Cav-1 expression increases during cellular differentiation and decreases upon dedifferentiation (Fan et al., 1983; Scherer et al., 1995; Koleske et al., 1995; Lee et al., 1998). It is also possible that the lipid raft environment differs in immature, nonmyelinating vs. differentiated, myelinating OLGs. Our observation that a subpopulation of OMgp is contained in lipid rafts is in keeping with data showing that $\sim 40 \%$ of CNP and MOG are associated with lipid rafts (Kim and Pfeiffer, 1999). It is hypothesized that the content of lipid rafts is dynamic, reflecting the activation/inactivation of various signaling pathways. The concentration of at least several proteins has been shown to fluctuate within lipid rafts or caveolae, depending upon cellular physiological state or presence/ absence of cross-linking (Mineo et al., 1999; Muller et al., 2002; Marta et al., 2003).

The physiological role of OMgp remains largely unknown. One proposed function is a growth suppressive effect (Habib et al., 1998a), and recently, the LRRs of OMgp have been shown to be important for mediating this 
property (Vourc'h et al., 2003). This finding is of interest, as the tumor suppressor protein neurofibromin is also growth suppressive (Johnson et al., 1994). The OMgp gene is contained within a large intron of the neurofibromatosis I gene (Viskochil et al., 1991) that encodes neurofibromin, and it has been suggested that this complex genetic arrangement may have evolved to enable coordinated expression of two proteins that influence related signaling pathways (Habib et al., 1998a). While the signaling components affected by OMgp have not been fully elucidated, our preliminary results show that OMgp and neurofibromin regulate similar signaling pathways such as mitogen-activated protein kinase and focal adhesion kinase, and transfection of OMgp into NIH-3T3 cells results in tyrosine phosphorylation of neurofibromin (M. Boyanapalli and D.D. Miko unpublished observations). OMgp has also been shown to be a potent inhibitor of neurite outgrowth (Kottis et al., 2002; Wang et al., 2002). Whether this has physiological relevance remains uncertain, although OMgp might possibly inhibit neuronal sprouting at the paranode, where OMgp appears to be localized (Mikol et al., 1999). OMgp is expressed relatively late in development, and its recently identified inhibitory effects on neurite outgrowth might simply represent extension of a physiological role in inhibiting sprouting of myelinated axons.

Given its ectomembrane structure, OMgp might interact with other molecules (Mikol et al., 1990b). Of note, co-immunoprecipitation of Omgp with Cav-1 (Fig. 6) likely reflects the presence of an as yet unidentified intermediary molecule, as OMgp is GPI-linked. Through its LRR or perhaps some other domain, it is possible that OMgp binds to (1) diffusible or matrix proteins, (2) $\operatorname{protein}(\mathrm{s})$ on other cells, or (3) protein(s) on the same cell. The latter is necessary if OMgp is involved in signal transduction pathways. GPI-linked proteins are thought to be present within lipid rafts in the vicinity of caveolin domains, and upon ligand binding, might become sequestered into caveolin-associated membrane domains (Kasahara and Sanai, 2000). Further, cross-linking of GPI-anchored proteins has been shown to activate signaling pathways, such as those involving src-family kinases (Brown, 1993; Mayor et al., 1994; Kasahara and Sanai, 2000). It is conceivable that, consequent to protein-protein interactions, OMgp becomes clustered, triggering signaling events within the OLG. At present, the only protein with which OMgp is known to interact is the Nogo receptor, a GPI-linked protein with LRRs that is expressed by neurons (Wang et al., 2002). It is unknown whether Nogo receptor binding induces clustering of OMgp.

OMgp is almost entirely membrane-associated, reflecting its GPI attachment. However, through the activation of an endogenous phospholipase, which might occur, for example, consequent to immune activation (Locati et al., 2001), OMgp could be released as a soluble form. Of the other identified neurite growth inhibitors, both MAG and the Nogo receptor might also be solubilized, the Nogo receptor via phospholipase, and MAG through proteolytic action. There is, in fact, some evidence for the latter (Sato et al., 1984). We hypothesize that membrane-associated OMgp, Nogo receptor and MAG might be focally released in certain disease states, particularly those associated with inflammation, such as multiple sclerosis. Soluble forms of these proteins might then have paracrine effects, resulting in inhibition of axonal regrowth.

In summary, we show that OMgp is a component of lipid rafts and in part associates with Cav-1, and that both are components of the myelin-axolemmal complex. We speculate that OMgp is a GPI-linked neural protein that interacts with Cav-1 and other unidentified membrane proteins and modulates signaling pathways at the myelin-axolemmal interface. Ongoing studies will further explore signaling pathways involving OMgp and the neural cell types affected.

\section{ACKNOWLEDGMENTS}

The authors thank Dr. N. Cashman for providing MO3.13 cells, and Drs. N. Groome, M. Rasband, and J. Salzer for providing antibodies. This work was supported by NIH NS42099-01 (to D.D.M.) and the Multiple Sclerosis Society of Canada (to P.E.B.). S. Bamri-Ezzine is the recipient of a studentship from the Multiple Sclerosis Society of Canada.

\section{REFERENCES}

Abrami L, Fivaz M, Kobayashi T, Kinoshita T, Parton RG, van der Goot FG. 2001. Cross-talk between caveolae and glycosylphosphatidylinositol-rich domains. J Biol Chem 276:30729-30736.

Aguirre et al. 2004. NG2-expressing cells in the subventricular zone are type C-like cells and contribute to interneuron generation in the postnatal hippocampus. J Cell Biol 165:575-89.

Ahmed SN, Brown DA, London E. 1997. On the origin of sphingolipid/ cholesterol-rich detergent-insoluble cell membranes: physiological concentrations of cholesterol and sphingolipid induce formation of a detergent-insoluble, liquid-ordered lipid phase in model membranes. Biochemistry 36:10944-10953.

Arvanitis DN, Wang H, Bagshaw RD, Callahan JW, Boggs JM. 2004. Membrane-associated estrogen receptor and caveolin-1 are present in central nervous system myelin and oligodendrocyte plasma membranes. J Neurosci Res 75:603-613.

Brown D. 1993. The tyrosine kinase connection: how GPI-anchored proteins activate T cells. Curr Opin Immunol 5:349-354.

Brown DA, London E. 1998. Functions of lipid rafts in biological membranes. Annu Rev Cell Dev Biol 14:111-136.

Chapman HA, Wei Y, Simon DI, Waltz DA. 1999. Role of urokinase receptor and caveolin in regulation of integrin signaling. Thromb Haemost 82:291-297.

Chang et al. 2000. NG2-positive oligodendrocyte progenitor cells in adult human brain and multiple sclerosis lesions. J Neurosci 20: 6404-6412.

Dawson et al. 2003. NG2-expressing glial progenitor cells: an abundant and widespread population of cycling cells in the adult rat CNS. Mol Cell Neurosci 24:476-488.

Fan JY, Carpentier J-L, Van Obberghen E, Grunfeld C, Gorden P, Orci L. 1983. Morphological changes of the 3T3-L1 fibroblast plasma membrane upon differentiation to the adipocyte form. J Cell Sci 61:219-230.

Fielding CJ, Fielding PE. 2003. Relationship between cholesterol trafficking and signaling in rafts and caveolae. Biochim Biophys Acta 1610:219-228.

Fra AM, Williamson E, Simons K, Parton RG. 1995. De novo formation of caveolae in lymphocytes by expression of VIP21-caveolin. Proc Natl Acad Sci USA 92:8655-8659.

Habib AA, Gulcher JR, Hognason T, Zheng L, Stefansson K. 1998a. The OMgp gene, a second growth suppressor within the NF1 gene. Oncogene 16:1525-1531. 
Habib AA, Marton LS, Allwardt B, Gulcher JR, Mikol DD, Hognason T, Chattopadhyay N, Stefansson K. 1998b. Expression of the oligodendrocyte-myelin glycoprotein by neurons in the mouse central nervous system. J Neurochem 70:1704-1711.

Hooper NM. 1999. Detergent-insoluble glycosphingolipid/cholesterolrich membrane domains, lipid rafts and caveolae (review). Mol Membr Biol 16:145-156.

Hooper NM, Turner AJ. 1988. Ectoenzymes of the kidney microvillar membrane. Differential solubilization by detergents can predict a glycosyl-phosphatidylinositol membrane anchor. Biochem J 250:865-869.

Johnson MR, DeClue JE, Felzmann S, Vass WC, Xu G, White R, Lowy DR. 1994. Neurofibromin can inhibit Ras-dependent growth by a mechanism independent of its GTPase-accelerating function. Mol Cell Biol 14:641-645.

Kasahara K, Sanai Y. 2000. Functional roles of glycosphingolipids in signal transduction via lipid rafts. Glycoconj J 17:153-162.

Kim T, Pfeiffer SE. 1999. Myelin glycosphingolipid/cholesterol-enriched microdomains selectively sequester the non-compact myelin proteins CNP and MOG. J Neurocytol 28:281-293.

Kobe B, Deisenhofer J. 1994. The leucine-rich repeat: a versatile binding motif. Trends Biochem Sci 19:415-421.

Kobe B, Deisenhofer J. 1995. Proteins with leucine-rich repeats. Curr Opin Struct Biol 5:409-416.

Koleske AJ, Baltimore D, Lisanti MP. 1995. Reduction of caveolin and caveolae in oncogenically transformed cells. Proc Natl Acad Sci USA 92:1381-1385.

Kottis V, Thibault P, Mikol D, Xiao Z-C, Zhang R, Dergham P, Braun PE. 2002. Oligodendrocyte-myelin glycoprotein (OMgp) is an inhibitor of neurite outgrowth. J Neurochem 82:1566-1569.

Kurzchalia TV, Parton RG. 1999. Membrane microdomains and caveolae. Curr Opin Cell Biol 11:424-431.

Laemmli UK. 1970. Cleavage of structural proteins during the assembly of the head of bacteriophage T4. Nature 227:680-685.

Lee SW, Reimer CL, Oh P, Campbell DB, Schnitzer JE. 1998. Tumor cell growth inhibition by caveolin re-expression in human breast cancer cells. Oncogene 16:1391-1397.

Lisanti MP, Tang ZL, Sargiacomo M. 1993. Caveolin forms a hetero-oligomeric protein complex that interacts with an apical GPI-linked protein: implications for the biogenesis of caveolae. J Cell Biol 123:595604.

Lisanti MP, Scherer PE, Tang Z-L, Sargiacomo M. 1994a. Caveolae, caveolin, and caveolin-rich membrane domains: a signalling hypothesis. Trends Cell Biol 4:231-235.

Lisanti MP, Scherer PE, Vicugiriene J, Tang Z-L, Hermanowski M, Vosatka A, Tu Y-H, Cook RF, Sargiacomo M. 1994b. Characterization of caveolin-rich membrane domains isolated from an endothelial-rich source: implications for human disease. J Cell Biol 126:111-126.

Lisanti MP, Tang Z-L, Scherer PE, Sargiacomo M. 1995. Caveolae purification and glycosylphosphatidylinositol-linked protein sorting in polarized epithelia. Methods Enzymol 250:655-668.

Liu J, Oh P, Horner T, Rogers RA, Schnitzer JE. 1997. Organized endothelial cell surface signal transduction in caveolae distinct from glycosylphosphatidylinositol-anchored protein microdomains. J Biol Chem 272:7211-7222.

Locati M, Riboldi E, Bonecchi R, Transidico P, Bernasconi S, Haribabu B, Morris AJ, Mantovani A, Sozzani S. 2001. Selective induction of phospholipase D1 in pathogen-activated human monocytes. Biochem J 358:119-125.

Marta CB, Taylor CM, Coetzee T, Kim T, Winkler S, Bansal R, Pfeiffer SE. 2003. Antibody cross-linking of myelin oligodendrocyte glycoprotein leads to its rapid repartitioning into detergent-insoluble fractions, and altered protein phosphorylation and cell morphology. J Neurosci 23:5461-5471.

Massimino ML, Griffoni C, Spisni E, Toni M, Tomasi V. 2002. Involvement of caveolae and caveolae-like domains in signalling, cell survival and angiogenesis. Cell Signal 14:93-98.

Mayor S, Rothberg KG, Maxfield FR. 1994. Sequestration of GPI anchored proteins in caveolae triggered by cross-linking. Science 264: 1948-1951.

McLaurin J, Trudel GC, Shaw IT, Antel JP, Cashman NR. 1995. A human glial hybrid cell line differentially expressing genes subserving oligodendrocyte and astrocyte phenotype. J Neurobiol 26:283293.

Menon K, Rasband MN, Taylor CM, Brophy P, Bansal R, Pfeiffer SE. 2003. The myelin-axolemmal complex: biochemical dissection and the role of galactosphingolipids. J Neurochem 87:995-1009.

Mikol DD, Alexakos MJ, Bayley CA, Lemons RS, Le Beau MM, Stefansson K. 1990a. Structure and chromosomal localization of gene for the oligodendrocyte-myelin glycoprotein. J Cell Biol 111:2673-2679.

Mikol DD, Gulcher JR, Stefansson K. 1990b. The oligodendrocyte-myelin glycoprotein belongs to a distinct family of proteins and contains the HNK-1 carbohydrate. J Cell Biol 110:471-479.
Mikol DD, Rongnoparut P, Allwardt BA, Marton LS, Stefansson K. 1993. The oligodendrocyte-myelin glycoprotein of mouse: primary structure and gene structure. Genomics 17:604-610.

Mikol DD, Hong H, Cheng H-L, Feldman EL. 1999. Caveolin-1 expression in Schwann cells. Glia 27:39-52.

Mikol DD, Scherer SS, Duckett SJ, Hong HL, Feldman EL. 2002. Schwann cell caveolin-1 expression increases during myelination and decreases following axotomy. Glia 38:191-199.

Mikol DD, Stefansson K. 1988. A phosphatidylinositol-linked peanut agglutinin-binding glycoprotein in central nervous system myelin and oligodendrocytes. J Cell Biol 106:1273-1279.

Mineo C, Gill GN, Anderson RG. 1999. Regulated migration of epidermal growth factor receptor from caveolae. J Biol Chem 274:3063630643 .

Muller G, Hanekop N, Wied S, Frick W. 2002. Cholesterol depletion blocks redistribution of lipid raft components and insulin-mimetic signaling by glimepiride and phosphoinositolglycans in rat adipocytes. Mol Med 8:120-136.

Norton WT, Poduslo SE. 1973. Myelination in rat brain: method of myelin isolation. J Neurochem 21:749-757.

Okamoto T, Schlegel A, Scherer PE, Lisanti MP. 1998. Caveolins, a family of scaffolding proteins for organizing "preassembled signaling complexes" at the plasma membrane. J Biol Chem 273:5419-5422.

Posnett DN, Tam JP. 1989. Multiple antigenic peptide method for producing antipeptide site-specific antibodies. Methods Enzymol 178: 739-746.

Sargiacomo M, Scherer PE, Tang Z, Kubler E, Song KS, Sanders MC, Lisanti MP. 1995. Oligomeric structure of caveolin: implications for caveolae membrane organization. Proc Natl Acad Sci USA 92:9407-9411.

Sargiacomo M, Sudol M, Tang Z-L, Lisanti MP. 1993. Signal transducing molecules and glycosyl-phosphatidylinositol-lined proteins form a caveolin-rich insoluble complex in MDCK cells. J Cell Biol 122:789-807.

Sato S, Yanagisawa K, Miyatake T. 1984. Conversion of myelin-associated glycoprotein (MAG) to a smaller derivative by calcium activated neutral protease (CANP)-like enzyme in myelin and inhibition by E-64 analogue. Neurochem Res 9:629-635.

Scherer PE, Tang Z, Chun M, Sargiacomo M, Lodish HF, Lisanti MP. 1995. Caveolin isoforms differ in their N-terminal protein sequence and subcellular distribution. J Biol Chem 270:16395-16401.

Schnitzer JE, McIntosh DP, Dvorak AM, Liu J, Oh P. 1995. Separation of caveolae from associated microdomains of GPI-anchored proteins. Science 269:1435-1439.

Schroeder R, London E, Brown D. 1994. Interactions between saturated acyl chains confer detergent resistance on lipids and glycosylphosphatidylinositol (GPI)-anchored proteins: GPI-anchored proteins in liposomes and cells show similar behavior. Proc Natl Acad Sci USA 91:12130-12134.

Sharom FJ, Lehto MT. 2002. Glycosylphosphatidylinositol-anchored proteins: structure, function, and cleavage by phosphatidylinositolspecific phospholipase C. Biochem Cell Biol 80:535-549.

Simons K, Toomre D. 2000. Lipid rafts and signal transduction. Nat Rev Mol Cell Biol 1:31-39.

Simons K, Wandinger-Ness A. 1990. Polarized sorting in epithelia. Cell 62:207-210.

Smith R, Braun PE. 1988. Release of proteins from the surface of bovine central nervous system myelin by salts and phospholipases. J Neurochem 50:722-729.

Song KS, Li S, Okamoto T, Quilliam LA, Sargiacomo M, Lisanti MP. 1996. Co-purification and direct interaction of Ras with caveolin, an integral membrane protein of caveolae microdomains. J Biol Chem 271:9690-9697.

Taylor CM, Coetzee T, Pfeiffer SE. 2002. Detergent-insoluble glycosphingolipid/cholesterol microdomains of the myelin membrane. J Neurochem 81:993-1004.

Trapp BD, Kidd GJ. 2004.Structure of the myelinated axon. In: Lazzarini RA, editor. Myelin biology and disorders. New York: Elsevier Science. p 3-27.

Viskochil D, Cawthon R, O'Connell P, Xu GF, Stevens J, Culver M, Carey J, White R. 1991. The gene encoding the oligodendrocyte-myelin glycoprotein is embedded within the neurofibromatosis type 1 gene. Mol Cell Biol 11:906-912.

Vourc'h P, Andres C. 2004. Oligodendrocyte myelin glycoprotein (OMgp): evolution, structure and function. Brain Res Brain Res Rev 45:115-124.

Vourc'h P, Moreau T, Arbion F, Marouillat-Vedrine S, Muh JP, Andres C. 2003. Oligodendrocyte myelin glycoprotein growth inhibition function requires its conserved leucine-rich repeat domain, not its glycosylphosphatidyl-inositol anchor. J Neurochem 85:889-897.

Wang KC, Koprivica V, Kim JA, Sivasankaran R, Guo Y, Neve RL, He Z. 2002. Oligodendrocyte-myelin glycoprotein is a Nogo receptor ligand that inhibits neurite outgrowth. Nature 417:941-944. 